\title{
SJP future, working from today
}

\author{
Mirko Antino \\ Universidad Complutense (Spain)
}

With the beginning of 2020 changes have taken place in SJP's editorial team. I am honored to be appointed as the new SJP chief editor, and I am pleased to welcome several new members of the editorial board. First of all, Dr. Alfredo Rodriguez-Muñoz, who enthusiastically accepted to take the charge of co-editor in chief, building a strongly committed managing team with Ana Montero and me. We together are sharing this new challenge, spending daily a nice amount of time in creatively drawing new editorial strategies, building on the amazing work done so far. We together welcome our new outstanding editorial board.

This is our first editorial note and we want to start illustrating our readers our plans for the future to continue improve and create new conditions for making SJP one of our favorite journals for publishing and reading high-quality psychological research. But, before entering in this new challenge, we want to acknowledge the work that has been done so far. First of all, by formally thank Dr. Javier Bandres Ponce, who devoted time, effort and personal care to the journal, interpreting the transformation of the discipline by pushing the journal further in its actual international dimension. He paid attention to the differential needs of everybody around, extending the culture of integrity and rigor of the previous editorial team (we take this chance to thank Dr. Juan Fernandez, without his first vision on this project, we would not be here now). Javier: if at the end of our term we have contributed to SJP to the same extent you did, we will truly feel we have made an enormous accomplishment.

Regarding our future plans, we will start refreshing the SJP mission: to promote the international dissemination of relevant empirical research and theoretical and methodological proposals in the various areas of specialization within psychology. To achieve that mission, and with the purpose of keep interpreting the evolution of the discipline, our interest is in publishing: a) empirical papers that regardless of their methodological orientation, either quantitative or qualitative,

Correspondence concerning this article should be addressed to Mirko Antino. Facultad de Psicología. Departamento de Psicobiología y Metodología en Ciencias del Comportamiento. Universidad Complutense. Campus de Somosaguas S/N, 28223 Pozuelo de Alarcón (Madrid).

E-mail: sjp.editor@ucm.es rigorously use their corresponding methods and properly justify their scientific approach. b) meta-analyses summarizing and integrating extant empirical findings; c) state-of-the-art reviews and theoretical papers that integrate previous research and propose innovative conceptual or theoretical frameworks, both articulating empirically testable propositions that stimulate further research on the field; d) methodological papers where new methods and measures are developed showing their added value to the comprehension and prediction of psychological phenomena.

Additionally, SJP is supporting the global movement towards a more transparent and reproducible psychological science and now is launching a special section that will accept three new types of articles: replications, registered reports and result-masked articles. Parallelly, with the finality of promoting critical debates among scholars, we will introduce a new section on "current debate in psychology". We will progressively introduce new initiatives and will keep our reader posted, updating our instructions for the authors. Finally, with the goal of improving the attractiveness of SJP to researchers, we will focus on maintaining and improving a good quality review process to serve our authors. Our goals are to offer timely and in-depth feedback. We therefore re-structured our review process, extending our editorial board. We will offer to our readers yearly updated data on the review time and our goals in this line.

To conclude this first communication, we will sincerely thank for all the support that our managing team received, both institutional (thanks to our Faculty Members and thanks to the Colegio Oficial de la Psicologia de Madrid for supporting this exciting project) and scientific (all those researchers and colleagues that generously spend their time in generating constructive and critical debates with us).

With the aim of keeping our doors open for further feedbacks and suggestion, we invite our readers to contact us at any moment! Now, let's go back to work!

How to cite this article:

Antino, M. (2020). SJP future, working from today. The Spanish Journal of Psychology, 23. e1. Doi:10.1017/SJP.2020.15 